\title{
Evolução do peso durante o primeiro ano de transplante renal e a ocorrência de Diabetes Mellitus após 5 anos de seguimento
}

\author{
Weight evolution during the first year of renal transplantation and the occurrence of \\ Diabetes Mellitus after 5 years of follow-up
}

\author{
Rafaela Siviero Caron Lienert ${ }^{1}$, Carlos Eduardo Poli de Figueiredo ${ }^{2}$, \\ Ana Elizabeth Prado Lima Figueiredo ${ }^{3}$
}

\begin{abstract}
RESUMO
Introdução: O período pós-transplante tardio é marcado por uma série de alterações nutricionais, os quais podem reduzir a sobrevida do órgão transplantado e do paciente.

Objetivo: Avaliar a evolução do peso durante o primeiro ano de transplante renal (TR) e o desenvolvimento de Diabetes Mellitus pós-transplante (DMPT) após cinco anos de seguimento.

Materiais e Métodos: Estudo de coorte retrospectiva, onde foram coletados dados de peso e outros parâmetros clínicos em prontuário do Setor de Nefrologia do Hospital São Lucas da PUCRS em Porto Alegre.

Resultados: Dos 35 pacientes estudados, ao final do primeiro ano, descrito como T12, observamos ganho de peso médio de $8,2 \%$, sendo que 29 deles $(82,9 \%)$ ganharam peso. O aumento torna-se significativo a partir do sexto mês após TR (T6) nas mulheres e após nove meses (T9) nos homens. O ganho de peso promove impacto sobre o IMC, apresentando diferença estatística entre dados iniciais (T0) $(23,2 \pm 4,3 \mathrm{~kg} / \mathrm{m} 2)$ e em T12 $(24,9 \pm 4,4 \mathrm{~kg} / \mathrm{m} 2)(p<0,001)$. Ao final do quinto ano, observa-se 6 óbitos $(17,1 \%)$ e $2(5,7 \%)$ perdas de enxerto. Dos 27 pacientes, observa-se incidência de $18,5 \%$ de DMPT, os quais apresentaram ganho de peso superior, apesar de não serem dados estatisticamente diferentes, e aumento acentuado durante o primeiro ano de TR (13,6\% do peso usual) em comparação com o grupo que não desenvolveu esta patologia (4,7\% do peso usual).

Conclusão: Observa-se aumento de peso após um ano de TR e, os pacientes que desenvolveram DMPT após 5 anos de seguimento apresentaram perfil de ganho de peso diferente do grupo que não desenvolveu a doença, apesar de serem dados sem diferença estatística.
\end{abstract}

Palavras-chave: transplante renal; ganho de peso; estado nutricional; doenças nutricionais e metabólicas.

\section{ABSTRACT}

Introduction: The late post-transplant period is characterizes by a series of nutritional changes, which can reduce organ and patient survival.

Objective: To evaluate the evolution of patient's weight during the first year after renal transplantation $(R T)$ and the occurrence of diabetes mellitus (DM) after 5 years of follow-up.

Materials and Methods: A retrospective cohort study was performed. Weight and other clinical data were collected in the records of the Department of Nephrology, São Lucas University Hospital of PUCRS in Porto Alegre.

Results: 35 patients were studied. At the end of the first year (data classified as T12), we observed an average weight gain of $8.2 \%$, while 29 (82.9\%) gained weight. The increase becomes significant from sixth month (T6) to ninth month (T9) in women and in men. The weight gain promotes impact on BMI, with significant difference between the beginning (TO) $(23.2 \pm 4.3 \mathrm{~kg} / \mathrm{m} 2)$ and the end of the first year after $R T(T 12)(24.9 \pm 4.4 \mathrm{~kg} / \mathrm{m} 2)(p<0.001)$. At the end of the fifth year, there were 6 deaths (17.1\%) and 2 (5.7\%) graft losses. Of the 27 patients, there was a 18.5\% incidence of DM, which showed higher weight gain, although not statistically different, and an increase during the first year of RT (13.6\% of usual weight) higher in comparison with the group that did not develop this condition (4.7\% of the usual weight).

Conclusions: There was an increase in weight after one year of RT, and the patients who developed DM after 5 years of follow-up showed a different weight gain profile, although data were not significant.

Keywords: renal transplantation; weight gain; nutritional status; nutritional and metabolic diseases.

\footnotetext{
${ }^{1}$ Nutricionista. Mestre e Doutoranda em Medicina e Ciências da Saúde pela Pontifícia Universidade Católica do Rio Grande do Sul (PUCRS).

${ }^{2}$ Médico. Doutor no University Department of Physiology/Jesus College da University of Oxford, Nephrology-Internal Medicine pela Renal Unit do Churchill Hospital em Oxford na Inglaterra. Professor Titular da Faculdade de Medicina e do Programa de PósGraduação em Medicina e Ciências da Saúde da PUCRS.

${ }^{3}$ Enfermeira. Doutora na Área de Concentração em Nefrologia do Programa de Pós-graduação em Clínica Médica e Ciências da Saúde da PUCRS. Professora Titular da Faculdade de Enfermagem, Nutrição e Fisioterapia da PUCRS.
} 


\section{INTRODUÇÃO}

O transplante renal (TR) é a terapia renal substitutiva (TRS) de escolha para pacientes com doença renal crônica (DRC) ${ }^{1}$, e, quando bem sucedido, proporciona melhor qualidade de vida em comparação com outros métodos dialíticos, contribuindo para o aumento da expectativa de vida e também beneficiando os pacientes nas esferas psicológica e emocional ${ }^{2-5}$. Esta modalidade de TRS apresenta números crescentes ${ }^{6}$. Atualmente, há em torno de 27.500 mil transplantados com enxertos renais funcionantes no país ${ }^{7}$. Porém, outros mais de 35.600 pacientes (aproximadamente 39\% dos pacientes em diálise) encontram-se em lista de espera, sendo, dessa forma, um número ainda insuficiente de procedimentos $^{8,9}$.

Esta terapia torna possível a prescrição de uma dieta menos restritiva ${ }^{10}$, porém não é livre de complicações nutricionais. No período de pós-transplante tardio - após as seis primeiras semanas da cirurgia - os efeitos colaterais do uso da imunossupressão promovem impacto no estado nutricional e, portanto, no prognóstico dos pacientes. Entre estes efeitos estão: hipercatabolismo protéico; diminuição da massa magra; hiperfagia; aumento dos depósitos de gordura, principalmente na região central; sedentarismo; obesidade; dislipidemia (DLP); intolerância à glicose, Diabetes Mellitus (DM) pós-transplante (DMPT) e Síndrome Metabólica $(\mathrm{SM})^{11}$. Nesta fase, observa-se também aumento da leptina sérica e este achado é frequentemente associado ao ganho de peso e à obesidade, o que acontece com maior intensidade no primeiro ano após o transplante, o que pode justificar o ganho de peso ainda mais acentuado nesta fase ${ }^{12}$.

O excesso de peso após TR, juntamente com outros achados - como alterações glicêmicas, aumento da lipemia e dos níveis pressóricos - parece acelerar a perda da função do rim e reduzir a efetividade do órgão transplantado $^{13}$. O agravo da doença pré-existente causado pela obesidade é consequência da diminuição do número de néfrons e da hiperfiltração induzida pelo excesso de peso ${ }^{14}$. Estudo de Gore et al. ${ }^{15}$ mostrou que a obesidade em receptores de TR está associada ao aumento do dano na função do enxerto e diminuição de sua sobrevida.
Considerando que diversos fatores afetam o peso após TR e esta alteração pode interferir na ocorrência de complicações aos pacientes, este estudo tem com objetivo avaliar a evolução do peso e outros parâmetros metabólicos durante $o$ primeiro ano de TR e o desenvolvimento de DMPT após cinco anos de seguimento.

\section{MATERIAIS E MÉTODOS}

O presente trabalho trata-se de uma coorte retrospectiva, sendo a coleta de dados realizada através dos registros em prontuário dos pacientes. Foram incluídos no estudo, todos os pacientes adultos (idade >18 anos) que receberam TR com a equipe de Nefrologia do Hospital São Lucas da Pontifícia Universidade Católica do Rio Grande do Sul (PUCRS) no ano de 2007. Critérios de exclusão: realização de transplante duplo (pâncreas-rim) ou transplante de outro órgão durante o período de acompanhamento e perda de seguimento por qualquer causa - como óbito, perda de enxerto, transferência de serviço ou falta de dados em prontuário.

Foram coletados dados clínico-demográficos (idade, sexo, doença de base, altura, modalidade e tempo de tratamento prévio ao transplante, tipo de doador, tempo de internação após TR), valores de peso, índice de massa corporal (IMC) e glicemia, prévios ao transplante (TO), durante a internação, e em um intervalo de 3 meses até completar 1 ano após o procedimento (classificados no texto como T3, T6, T9, T12, trimestralmente após o TR). Foram avaliados semestralmente resultados de exames séricos de colesterol total (CT) e lipoproteína de alta densidade (HDL-c), triglicerídeos (TG), conforme disponível nos prontuários. Nestes casos, foi utilizada a primeira medida do primeiro semestre e a última medida do segundo semestre após o TR ou a única medida de cada semestre. $O$ cálculo do IMC foi realizado de acordo com a seguinte fórmula: IMC = peso $(\mathrm{kg}) /$ altura $\left(\mathrm{m}^{2}\right)$, e a sua classificação foi feita conforme a Organização Mundial de Saúde ${ }^{16}$. A fim de observar o impacto da evolução do peso no desfecho DMPT, foram acompanhados, também de forma retrospectiva, os mesmos pacientes nos cinco anos após o TR. 
Os dados estão apresentados como média \pm desvio padrão, medianas e percentis, dependendo da característica de normalidade dos mesmos. As análises de normalidade e variabilidade foram realizadas através do teste de Kolmogorov-Smirnov, as comparações das médias dos dados pareados foram realizadas a partir do teste t pareado e as frequências comparadas por teste de Wilcoxon. Diferenças com valores de $p \leq 0,05$ foram consideradas significativas, através do software Statistical Package for the Social Sciences, versão 17,0.

Este estudo foi aprovado pelo Comitê de Ética em Pesquisa da PUCRS, conforme número do protocolo CEP 09/04533.

\section{RESULTADOS}

Dos pacientes 55 pacientes transplantados incluídos no estudo, $5(9,1 \%)$ apresentaram perda de enxerto, sendo que destes casos, 3 pacientes evoluíram a óbito. Observa-se, também, outros 2 óbitos não relacionados à perda do enxerto, compreendendo 5 óbitos $(9,1 \%)$ e 6 perdas de seguimento por transferência de serviço de acompanhamento e outros 7 sem dados de peso suficientes em prontuário. As causas de exerese do enxerto compreenderam trombose e ruptura renal. Já as causas de óbitos não relacionadas à perda do enxerto foram infarto agudo do miocárdio e estrangulamento de hérnia intestinal. Portanto, o número de pacientes incluídos no estudo foi de 35 pacientes. As características clínicas e demográficas dos pacientes estudados estão apresentadas na tabela 1 .

Após 1 ano de transplante, os pacientes apresentaram ganho de peso médio de $8,2 \%$. Observou-se que apenas $6(17,1 \%)$ pacientes apresentaram estabilidade ou perda de peso durante 0 primeiro ano pós-TR, enquanto que 29 (82,9\%) ganharam peso, sendo que 15 (42,9\%) apresentaram ganho de peso superior a $10 \%$ do peso pré-TR e mais de $4(11,4 \%)$ ganho de peso superior a $20,0 \%$.

Observa-se que o aumento de peso já é diferente significativamente a partir de T3 na amostra total. Na análise separada por gênero, observa-se que este aumento torna-se significativo a partir de T6 nas mulheres e em T9 nos homens (tabela 2). Como foram dados coletados retrospectivamente, ouve uma perda
TABELA 1 - Descrição clínico-demográfica da amostra em estudo $(n=35)$.

\begin{tabular}{|c|c|}
\hline Variáveis & $\begin{array}{r}\text { Total da } \\
\text { Amostra } \\
\end{array}$ \\
\hline Idade (anos) (média $\pm D P)$ & $42,6 \pm 16,2$ \\
\hline Sexo Feminino (n/\%) & $17(48,6 \%)$ \\
\hline Peso Pré-TR (kg) (média $\pm D P)$ & $64,3 \pm 14,6$ \\
\hline IMC Pré-TR (kg/m²) (média $\pm D P)$ & $23,2 \pm 4,3$ \\
\hline \multicolumn{2}{|l|}{ Doença de Base } \\
\hline HAS & $9(25,7 \%)$ \\
\hline DM & $2(5,7 \%)$ \\
\hline DRP & $4(11,4 \%)$ \\
\hline LES & $2(5,7 \%)$ \\
\hline Infecções Crônicas & $3(7,3 \%)$ \\
\hline Outras causas & $19(46,3 \%)$ \\
\hline $\begin{array}{l}\text { Tempo de Tratamento Prévio ao TR } \\
\text { (meses) (med/P25-P75) }\end{array}$ & $36(12-93)$ \\
\hline Doador Falecido & $28(68,3 \%)$ \\
\hline \multicolumn{2}{|l|}{ Modalidade de Tratamento Prévio ao TR } \\
\hline Conservador & $1(2,9 \%)$ \\
\hline Hemodiálise & $28(80 \%)$ \\
\hline Diálise Peritoneal & $6(17,1 \%)$ \\
\hline $\begin{array}{l}\text { Tempo de Internação após TX } \\
\text { (dias) (med/P25-P75) }\end{array}$ & $19,0(9,0-30,3)$ \\
\hline
\end{tabular}

n: número; DP: desvio padrão; TR: transplante renal; $\mathrm{kg}$ : quilograma; HAS: hipertensão arterial sistêmica; DM: diabetes mellitus; DRP: doença renal policística; LES: lúpus eritematoso sistêmico; TR: transplante renal.

relacionada à falta de registro da altura de um paciente, totalizando a análise relacionada ao IMC de 34 pacientes. O ganho de peso promove impacto sobre 0 IMC, apresentando diferença estatística entre T0 $(23,2 \pm 4,3 \mathrm{~kg} / \mathrm{m} 2)$ e T12 $(24,9 \pm 4,4 \mathrm{~kg} / \mathrm{m} 2)(\mathrm{p}<0,001)$. O percentual de pacientes conforme classificação do IMC (baixo peso, eutrófico, sobrepeso e obeso) no período prévio ao transplante (T0) e ao final do seguimento (T12) está apresentado na (tabela 3) e quando submetidos a análise estatística através do teste de Wilcoxon não apresentou diferença $(p=0,405)$. Os níveis glicêmicos começam a apresentar diminuição significativa em T9 $(92,7 \pm 38,2 ; \quad p=0,049) \quad$ e permaneceram diminuída até T12 $(90,7 \pm 26,9 ; p=0,039)$, assim como o CT $(p=0,041)$ entre 0 primeiro $(215,18 \pm 54,8)$ e o segundo semestre $(198,5 \pm 67,0)$ de avaliação. Este achado se dá possivelmente pelo controle médico e medicamentoso adequado. Os dados 
TABELA 2 - Peso trimestral durante o primeiro ano após transplante renal.

\begin{tabular}{lccccc}
\hline Peso & T0 & T3 & T6 & T9 & T12 \\
\hline Total $(\mathbf{n = 3 5 )}$ & $64,3 \pm 14,7$ & $66,2 \pm 14,0^{*}$ & $68,0 \pm 14,96^{*}$ & $69,1 \pm 15,4 \#$ & $68,9 \pm 14,9 \#$ \\
Homens $(\mathbf{n = 1 8})$ & $68,813,9$ & $70,4 \pm 13,9$ & $72,2 \pm 14,6$ & $74,2 \pm 15,3^{*}$ & $73,2 \pm 15,6^{*}$ \\
Mulheres $(\mathbf{n = 1 7})$ & $60,3 \pm 14,9$ & $62,6 \pm 13,2$ & $64,3 \pm 14,7^{*}$ & $64,7 \pm 14,2^{*}$ & $65,2 \pm 13,4^{*}$ \\
\hline
\end{tabular}

IMC: índice de massa corporal; $n$ : número. Dados com distribuição normal estão expressos como média \pm desvio padrão. ${ }^{*} \mathrm{p}<0,05$ vs. T0, $\# p<0,001$ vs. T0.

de HDL-c e TG não apresentaram diferença estatística durante o período do estudo (tabela 4).

Os mesmos pacientes foram acompanhados retrospectivamente no decorrer dos quatro anos seguintes, sendo constatados outros 6 óbitos $(17,1 \%)$ e $2(5,7 \%)$ perdas de enxerto, totalizando, ao final do quinto ano, uma amostra de 27 pacientes. Quando se observa 0 perfil dos pacientes que perderam seguimento até o final do quinto ano (tanto por óbito ou por perda de enxerto), percebe-se média de ganho de peso em T12 de $12,5 \pm 8,8 \mathrm{~kg}$, sendo que todos eles apresentaram ganho de peso neste período.

Apesar de não serem dados estatisticamente diferentes, percebe-se que o grupo que desenvolveu DMPT neste seguimento, apresentou, além de um ganho de peso superior, aumento de peso acentuado durante o primeiro ano de TR (13,6\% do peso usual) em comparação com o grupo que não desenvolveu esta patologia associada ( $4,7 \%$ do peso usual), sendo, ainda, que o peso no período pré-transplante é menor no grupo afetado pela doença (figura 1).

\section{DISCUSSÃO}

Os principais dados resultantes de nosso estudo são referentes ao aumento de peso após um ano de TR, o que se observa em ambos os sexos, inclusive com alteração da classificação de IMC dos pacientes. Observa-se que os sujeitos que desenvolveram DMPT após 5 anos de seguimento não apresentaram diferença estatística no que se refere ao padrão de ganho de peso. Considerações estas que salientam a necessidade de mais estudos que viabilizem a análise de um número maior de pacientes com a doença, por acreditarmos que os acontecimentos metabólicos durante o primeiro ano após o procedimento possui um fator importante no prognóstico dos pacientes.

O TR representa o tratamento de escolha para DRC em estágio terminal ${ }^{17}$. Com o desenvolvimento de novas estratégias imunossupressoras, a incidência de rejeição aguda diminuiu drasticamente, aumentando a sobrevida do enxerto ${ }^{17}$. O rim é o órgão sólido mais transplantado e o transplante bem sucedido promove restauração da função renal, porém algumas desordens metabólicas podem persistir e outras podem surgir ${ }^{18}$. Segundo dados do Registro Brasileiro de Transplante da Associação Brasileira de Transplante de Órgãos (ABTO/RBT), a expectativa de vida nesta população é promissora, a sobrevida atual do paciente transplantado renal varia de 91 para doador falecido (DF) e 97,0\% para doador cadáver (DV) e, do enxerto varia de 83,0 a 92,0\% (DF e DV, respectivamente) no primeiro ano após o procedimento $^{18}$.

O IMC acima da normalidade constitui um fator de risco importante para morbimortalidade da população em geral, porém também é associado ao aumento da sobrevida de pacientes em diálise ${ }^{17}$. O efeito da obesidade em transplantados renais ainda é controverso, porém sabe-se que é um achado frequente nestes pacientes e um fator de risco para HAS, doença coronariana, alterações nos lipídicos séricos e no desenvolvimento de $\mathrm{DMPT}^{19,20}$. A segunda principal causa de morte em pacientes transplantados são as doenças cardiovasculares $(29,0 \%)$, atrás apenas das infecções $(50,0 \%)$, e aproximadamente $50,0 \%$ dos receptores irão apresentar algum tipo de evento cardiovascular em 15 anos de $\mathrm{TR}^{18,21}$.

Estudo que analisou resultados após TR, mostrou que no primeiro ano, o ganho de peso entre $10,0 \%$ a $19,9 \%$ estaria associado com melhores resultados. Já no segundo ano, a estabilidade do peso ou um pequeno aumento também contribuiriam positivamente. Porém, nos primeiros dois anos após o procedimento, a perda de peso ou o aumento excessivo ( $\geq 20,0 \%$ em 1 ano e $\geq 10,0 \%$ em 2 anos) estão associados a maior 
TABELA 3 - Distribuição dos pacientes segundo classificação do estado nutricional pelo IMC antes e após 1 ano de transplante renal $(n=34)$.

\begin{tabular}{lcc}
\hline IMC $\left(\mathbf{k g} / \mathbf{m}^{2}\right)$ & $\begin{array}{c}\text { Antes do } \\
\text { Transplante Renal } \\
(\mathbf{n} / \%)\end{array}$ & $\begin{array}{c}\text { Após 1 ano de } \\
\text { Transplante Renal } \\
(\mathbf{n} / \%)\end{array}$ \\
\hline Baixo Peso & $1(3,0)$ & $2(5,9)$ \\
Eutrófico & $20(58,8)$ & $18(52,9)$ \\
Sobrepeso & $10(29,4)$ & $8(23,5)$ \\
Obesidade & $3(8,8)$ & $6(17,7)$ \\
Total & $34(100)$ & $34(100)$ \\
\hline
\end{tabular}

IMC: índice de massa corporal. Teste de Wilcoxon comparando os percentuais antes e após o transplante renal $(p=0,405)$.

mortalidade. A média de idade dos transplantados avaliados em nosso estudo é de 42 anos, porém o grupo com maior ganho de peso (>20,0\%) é mais jovem, com média de idade de 31 anos, dado este que é também foi constatado por Chang em $2008^{11}$.

Este mesmo autor constatou em outro estudo que, aproximadamente, $75,0 \%$ dos pacientes abaixo do peso ideal apresentaram IMC normal ou acima da normalidade do primeiro ao quinto ano pós-transplante, o que sugere uma mudança significativa do peso sustentada $^{14}$. Em nosso estudo, 19,0\% dos eutróficos passaram a ser classificados como sobrepeso e $36,2 \%$ dos sobrepesos passaram a ser classificados como obesos ainda no primeiro ano.

A obesidade aumenta a demanda metabólica do rim, porém o número de néfrons não aumenta como o ganho de peso, o que faz com que a filtração glomerular e o fluxo plasmático renal se elevem para compensar esse incremento do metabolismo. A obesidade pode ser um iniciador ou acelerador do dano renal, contribuindo para diminuição do número de néfrons em pacientes não transplantados ${ }^{14}$. Após TR, o excesso de peso parece acelerar a perda da função do rim. O agravo da doença pré-existente causado pela obesidade é consequência da diminuição do número de néfrons e da hiperfiltração induzida pelo excesso de peso ${ }^{14}$. Estudo de Gore et al. ${ }^{15}$ mostrou que a obesidade em receptores de TR está associada ao aumento do dano na função do enxerto e diminuição de sua sobrevida.

A SM, caracterizada pela combinação de obesidade central, dislipidemia, hipertensão, resistência à insulina, é observada em pacientes após TR, a qual tem sua

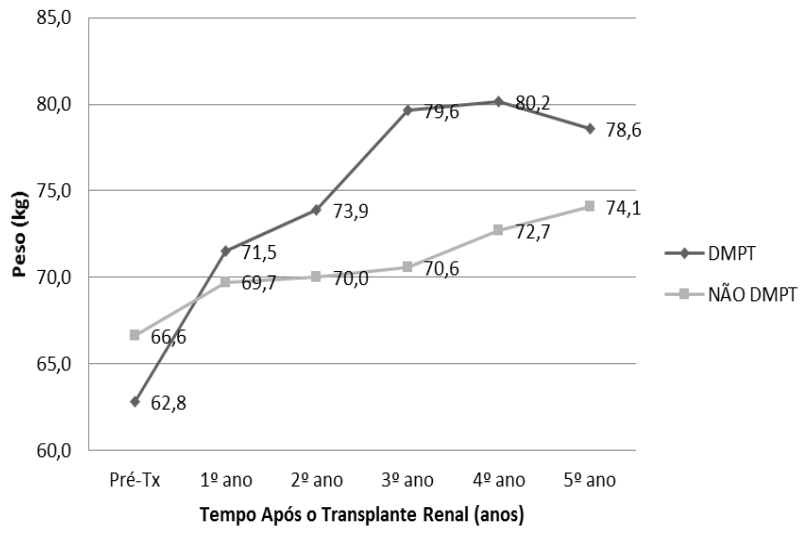

FIGURA 1 - Evolução do peso em um seguinto de 5 anos após transplante renal e desenvolvimento de Diabetes Mellitus no decorrer do período. DMPT: Diabetes Mellitus pós-transplante.

prevalência aumentada com 0 ganho de peso acentuado. Nesta população, a SM também está associada à doença cardiovascular, DMPT, deterioração da função do enxerto e a sua perda, sendo fundamental o controle dietético para evitar estas complicações ${ }^{22,23}$.

O aumento global da prevalência de obesidade eleva o número de pacientes obesos candidatos a transplantes ${ }^{24}$. A prevalência de obesidade em nossa amostra foi de 8,8\%, aumentando progressivamente no decorrer do primeiro ano de TR, até 17,7\%. O aumento ponderal parece permanecer até 20 anos após TR, em um padrão progressivo, porém com maior intensidade durante o primeiro ano ${ }^{5,11,25}$. Conforme nossos achados, o maior aumento de peso se dá até o 9 mês após o TR, sendo nos primeiros 6 meses nas mulheres e até o final do terceiro trimestre nos homens. Coroas e colaboradores sugerem que possa haver diferenças no padrão da composição corporal entre os gêneros após TR, pois observaram, em dois estudos de 3 meses de acompanhamento, que homens não recuperaram os parâmetros antropométricos, apresentando maior quantidade de água corporal enquanto que as mulheres iniciam mais próximas do grupo sadio e ganham peso e massa gorda neste mesmo período ${ }^{25,26}$.

O ganho de peso após o TR não é necessariamente um indicativo negativo, em muitos pacientes, este aumento caracteriza recuperação de um estado nutricional prejudicado prévio a esta terapia. Porém, o que se observa é que este aumento é dependente de massa gorda, elevando a lipemia e a resistência à insulina $^{25}$. Sabe-se que a obesidade continua sendo um desafio aos profissionais de saúde envolvidos no 
TABELA 4 - Comparação dos exames laboratoriais avaliados no decorrer de 1 ano após o Transplante Renal.

\begin{tabular}{|c|c|c|c|c|c|c|}
\hline Exames Laboratoriais & TO & T3 & T6 & T9 & T12 & $\mathbf{P}$ \\
\hline CT (mg/dL) & - & \multicolumn{2}{|c|}{$215,18 \pm 54,8$} & \multicolumn{2}{|c|}{$198,5 \pm 67,0$} & 0,041 \\
\hline HDL-c (mg/dL) & - & \multicolumn{2}{|c|}{$52,0 \pm 13,4$} & \multicolumn{2}{|c|}{$48,0 \pm 11,3$} & 0,109 \\
\hline $\mathrm{TG}(\mathrm{mg} / \mathrm{dL})$ & - & \multicolumn{2}{|c|}{$228(30-978)$} & \multicolumn{2}{|c|}{$193,5(90-646)$} & 0139 \\
\hline Glicemia (mg/dL) & - & $94,7 \pm 22,6$ & $96,8 \pm 51,9$ & $92,7 \pm 38,2$ & $90,37 \pm 26,9$ & 0,039 \\
\hline
\end{tabular}

CT: colesterol total; HDL-c: lipoproteína de alta densidade; TG: triglicerídeos; mg: miligrama; dL: decilitro. Dados com distribuição normal estão expressos como média \pm desvio padrão e dados não paramétricos como mediana e intervalo interquartil.

cuidado de transplantados renais, pois é preciso controlar o ganho de peso excessivo e a velocidade deste incremento, evitando efeitos sobre o enxerto e o desenvolvimento de patologias associadas ${ }^{27}$. O ganho de peso contribui para a ocorrência de fatores de risco não-imunológicos que podem levar ao desenvolvimento de disfunção crônica do enxerto ${ }^{28}$. Um estudo que avaliou o impacto da redução calórica e protéica em pacientes com aumento do IMC após TR mostrou que após 12 meses a intervenção na dieta proporcionou retorno ao IMC normal em todos pacientes $\left(<25,0 \mathrm{~kg} / \mathrm{m}^{2}\right)^{29}$. Segundo estudos de intervenção dietética, a abordagem nutricional adequada é capaz de proporcionar balanço metabólico associado à redução de peso e dos níveis séricos de CT, TG e glicose, assim como evitam ganho de peso excessivo após $\mathrm{TR}^{30}$.

A resistência a insulina é comum nesta população $(31,0 \text { a } 33,0 \%)^{31}$. O aparecimento de DMPT, definida por glicemia $\geq 126,0 \mathrm{mg} / \mathrm{dL}$, acontece em $2,0 \%$ a $53,0 \%$ dos receptores e suas manifestações clínicas vão desde a hiperglicemia assintomática até a cetoacidose ${ }^{32}$. A glicemia de nossos pacientes diminui com o passar dos meses após TR. Por se tratar de dados retrospectivos, acredita-se que este achado tenha ocorrido em função do acompanhamento médico constante, mudança de imunossupressores e do uso de terapia medicamentosa assim que necessário, evitando glicemias elevadas após o diagnóstico de DMPT. Assim como a diminuição significativa do CT, essa diminuição pode ser justificada pelo controle clínico e medicamentoso de qualidade e, também, pela redução das doses de imunossupressão no segundo semestre após TR.

Os fármacos de imunossupressão podem ter interferência direta no perfil glicêmico e aumento do risco de DMPT. O uso de terapia imunossupressora com esteróides e inibidores da calcineurina, especialmente tacrolimus, aumenta a resistência à insulina e a gliconeogênse hepática, favorecendo ao desenvolvimento desta complicação. O uso de tacrolimus bloqueia o gene de transcrição da insulina humana, resultando na diminuição da sua secreção e resistência periférica. Outros fatores associados ao uso destes medicamentos - como gênero, idade, tipo de doador e presença de infecções - aumentam o risco de desenvolvimento de $\mathrm{DMPT}^{32,33}$

Existem diversas limitações presentes em nosso estudo. A primeira refere-se ao delineamento: por se tratar de um estudo retrospectivo, realizado com dados de prontuário, verificamos que os existem muitos dados falhos que deveriam ser melhor registrados pelos profissionais de saúde envolvidos no cuidado dos pacientes em vista de estudos com maior validade; para uma avaliação mais precisa destes dados, uma coorte prospectiva possibilitaria maior controle dos dados. A segunda refere-se ao tamanho amostral, qual é pouco representativo, porém válido para reflexão da prática clínica e planejamento de um estudo melhor conduzido. A terceira refere-se aos dados de avaliação nutricional, os quais poderiam, se realizados de maneira prospectiva, ser mais completos, englobando a avaliação criteriosa da composição corporal. A quarta é referente ao controle do uso de drogas, onde poderia ser incluído critério de inclusão de pulsoterapia ou outra intervenção que pudesse interferir no ganho de peso agudamente.

São necessários estudos prospectivos desenhados para observação da composição corporal após TR, a fim de compreender melhor os mecanismos envolvidos no 
ganho de peso e nas alterações metabólicas após TR para que estratégias nutricionais eficazes sejam realizadas, promovendo benefícios a esta população.

\section{REFERÊNCIAS}

1. Hariharan S, McBride A, Bennett LA, Cohen EP. Risk factors for renal allograft survival from older cadaver donors. Transplantation 1997; 64(12):1748-54.

2. Jezior D, Krajewska M, Madziarska K, Kurc-Darak B, Janczak D, Patrzalek D, Borysławski K, Klinger M.Posttransplant Owerweight and Obesity: myth or reality? Transplant Proc 2007; 39(9):2772-5.

3. Elster EA, Leeser DB, Morrissette C, Papek JM, Quiko A, Hale DA, Chamberlain C, Salaita C, Kirk AD, Mannon RB. Obesity following kidney transplantation and steriod avoidance immunosupression. Clin Transplant 2008; 22(3):354-9.

4. Fonseca AG, Mignone SC, Tuma da Ponte JR, Cruz AS, Souza FJ, Toscano PM, Ferreira de Almeida M, Lima SM. Análise dos resultados iniciais do programa de transplante renal intervivos no Estado do Pará. ABTO 2006; 10(1):525-9.

5. Hricik DE. Metabolic syndrome in kidney transplantation. Clin J Am Soc Nephrol 2011; 6(7):1781-5.

6. Hariharan S, Johnson CP, Bresnahan BA, Taranto SE, McIntosh MJ, Stablein D. Improved graft survival after renal transplantation in the United States, 1988 to 1996. N Engl J Med 2000; 342(9):605-12.

7. Lugon JR. Doença renal crônica no Brasil: um problema de saúde pública. J Bras Nefrol 2009; 31(1):2-5.

8. Wolf RA, Ashby VB, Milford EL, Ojo AO, Ettenger RE, Agodoa LY, Held PJ, Port FK. Comparison of mortality in all patients on dialysis, patients on dialysis awaiting transplantation, and recipients of a first cadaveric transplant. N Engl J Med 1999; 341(23):1725-30.

9. Sesso RC, Lopes AA, Thome FS, Lugon JR, Santos DR. Dialysis Census Results - 2010 from the Brazilian Society of Nephrology J Bras Nefrol 2011; 33(4):442-7.

10. Silva DS, lanhez LE. Characterization of the sample of patients under care in the Renal Transplant Unit of the Division of Urologic Clinic of HCFMUSP along two months. J Bras Transpl 2006; 9:525-9.

11. Chang SH, McDonald SP. Post-Kidney transplant weight change as marker of poor survival outcomes. Transplant 2008; 85(10):1443-3.

12. Souza GC, Costa C, Scalco R, Gonçalves LV, Manfro RC. Serum leptin, insulin resistance, and body fat after renal transplantation. J Ren Nutr 2008;8(6):479-88.

13. Stacey Phillips S, Heuberger R. Metabolic disorders following kidney transplantation. J Ren Nutr 2012; 22(5):451-60.

14. Kramer $\mathrm{H}$, Luke A. Obesity and kidney disease: a big dilemma. Curr Opin Nephrol Hypertens 2007; 16(3):23741.

15. Gore JL, Pham PT, Danovitch GM, Wilkinson AH, Rosenthal JT, Lipshutz GS, Singer JS. Obesity and outcome following renal transplantation. Am J Transplant 2006; 6(2):357-63.

16. Food and Agriculture Organization/World Health Organization. Physical Status: The use and interpretation of antropometry. Geneva, WHO, 1995 (WHO, offset publication, 854).

17. Guida B, Trio R, Laccetti R, Laccetti R, Nastasi A, Salvi E, Perrino NR, Caputo C, Rotaia E, Federico S, Sabbatini M. Role of dietary intervention on metabolism abnormalities and nutricional status after renal transplantation. Nephrol Dial Transplant 2007;22(11):3304-10.

18. Associação Brasileira de Transplante de Órgãos. Registro Brasileiro de Transplantes 2011; XVII(4):1-19. [acessado 2012 jul. 4]. Disponível em: http://www.abto.org.br/abtov02/portugues/populacao/rbt.

19. Marceón R, Fernández J, Pascual J, Teruel JL, Villafruela JJ, Rodriguez N, Martins J, Burgos FJ, Ortuño J. High body massa index and posttransplant weight gain are not risk factors for kidney graft and patient outcome. Transpl Proc 2007; 39(7):2205-7.

20. Kent PS. Issues of obesity in kidney transplantation. J Ren Nutr 2007; 17(2):107-13.

21. Ward HJ. Nutritional and metabolic issues in solid organ transplantation: targets for future research. J Ren Nutr 2009; 19(1):111-22.

22. Hricik DE. Metabolic syndrome in kidney transplantation: management of risk factors. Clin J Am Soc Nephrol 2011; 6(7):1781-5.

23. Teixeira APSF, Fernandes NMS, Mata GF, Chaoubah A Paula RB, Bastos MG. Prevalence of metabolic syndrome in renal transplantation. J Bras Nefrol 2012; 34(1):16-21.

24. Hasse J. Pretransplant obesity: a weighty issue affecting transplant candidacy and outcomes. Nutr Clin Pract 2007; 22(5):494-504.

25. Coroas A, Oliveira JG, Sampaio S, Borges C, Tavares I, Pestana M, Almeida MD. Nutritional status and body composition evolution in early post-renal transplantation: is there a female advantage? Transplant Proc 2005; 37(6):2765-70.

26. Coroas ASPS, Oliveira JGG, Sampaio SMM, Tavares IC, Pestana M, Almeida MD. Postrenal Transplantation Body Composition: Different Evolution Depending on Gender. J Ren Nutr 2007; 17(2):151-6.

27. Armstrong KA, Campbell SB, Hawley CM, Johnson DW, Isbel NM. Impact of obesity on renal transplant outcomes. Nephrology (Carlton) 2005; 10(4):405-13.

28. Kalantar-Zadeh K, Abbott KC, Salahudeen AK,Kilpatrick RD, Horwich TB. Survival advantages of obesity in dialysis patients. Am J Clin Nutr 2005; 81(3):543-54.

29. Bellinghieri G, Bernadri A, Piva M, Pati T, Stoppa F, Scaramuzzo P, Garizzo O, Santoro D, Savica V, Bucciante G. Metabolic syndrome after kidney transplantation. J Ren Nutr. 2009; 19(1):105-10.

30. Jezior D, Krajewsk K, Madziarska B, Regulska-llow B, llow $\mathrm{R}$, Jańczak $\mathrm{D}$, Patrzałek $\mathrm{D}$, Klinger M. Weigth Rediction in Renal Transplant Recipients Program: the first successes. Transpl Proc 2007; 39(9):2769-71.

31. Teplan V, Valkovsky I, Teplan VJ, Stollova M, Vyhnanek F, Andel M. Nutritional consequences of renal transplantation. J Ren Nutr 2009; 19(1):95-100.

32. Mazzali F, Lalli A, Alves-Filho G, Mazzali M. Posttransplant Diabetes Mellitus: incidence and risk factors. Transplant Proc 2008; 40(3):764-6.

33. Rodrigo $E$, de Cos MA, Fernández-Fresnedo $G$, Sanchez B, Ruiz JC, Piñera C, Palomar R, Cotorruelo JG, GómezAlamillo C, de Castro SS, de Francisco AL, Arias M. Higher Initial Tacrolimos Blood Levels and ConcentrationDose Rations in Kidney Transplant Recipient Who Develop Diabetes Mellitus. Transplant Proc 2005; 37(9):3819-20.

\section{Endereço para correspondência:}

Rafaela Siviero Caron Lienert Av. Ipiranga $n^{\circ} 6690$

Porto Alegre/RS - CEP 90610-000

Telefone: +55 5133391211

E-mail: rafaelacaron@terra.com.br 